\title{
A case of acute pericarditis and subsequent pericardial effusion in COVID-19 patient: a case report
}

\author{
Waddah Osman ${ }^{1}$, Abdelmuniem Ahmed ${ }^{1}$, Omer Elawad $^{1}$, and Ahmed Albashir ${ }^{1}$ \\ ${ }^{1}$ University of Gezira Faculty of Medicine
}

September 10, 2020

\begin{abstract}
Acute pericarditis and pericardial effusion are rare extrapulmonary presentations of COVID-19. They can occur without concomitant pulmonary disease or myocardial injury. A high index of suspicion is necessary to assure early diagnosis and treatment.
\end{abstract}

\section{Keywords:}

Covid-19, acute pericarditis, pericardial effusion, case report.

Key Clinical Message Acute pericarditis and pericardial effusion are one of the rare extra-pulmonary presentations of COVID-19. They can occur without concomitant pulmonary disease or myocardial injury. Studies are needed to determine the exact mechanism of acute pericarditis and pericardial effusion in COVID19 patients and the optimal therapeutic strategies.

\section{Introduction:}

Coronavirus disease 2019 (COVID-19) is a current pandemic outbreak caused by a novel coronavirus now called severe acute respiratory syndrome coronavirus 2 (SARS-CoV-2). The commonest cardiac complications include acute myocardial injury, arrhythmias, acute myocarditis and severe left ventricular dysfunction. ${ }^{1}$ Conversely, reports of pericarditis and pericardial effusion are rare.

A large pericardial effusion due COVID-19 has been reported in a 41 woman in Saudi Arabia. ${ }^{2}$ Besides, cardiac tamponade secondary to COVID-19, ${ }^{3}$ life-threatening cardiac tamponade complicating myopericarditis in COVID-19, ${ }^{4}$ acute myopericarditis with pericardial effusion and cardiac tamponade in a patient with COVID-19, ${ }^{5}$ and a case of acute pericarditis and cardiac tamponade in a patient with COVID-19 have all been reported. ${ }^{6}$

Hereby, we describe a case of acute pericarditis and pericardial effusion in a COVID-19 patient.

\section{Case presentation:}

A previously healthy 50-years old Sudanese man with no prior significant medical history came to a university hospital, Sudan, complaining of chest pain, stabbing in nature, increased in severity when supine position and improved with leaning forward. He denied any history of cough or shortness of breath.

During the physical examination, the patient preferred sitting leaning forward, not dyspneic. Her BP was 160/80, pulse rate 96 regular, temperature 38.1 and oxygen saturation $96 \%$ on room air. The JVP was not raised. Pericardial friction rub was not heard. Cardiovascular and chest examination was unremarkable.

Initial laboratory investigations revealed the following: WBCs $11.0 \times 109 / \mathrm{L}(4.0-11.0 \times 109 / \mathrm{L})$, lymphopenia $5 \%$, ESR 50mm / hour, The liver and kidney function were within normal values. D-dimer $350 \mathrm{ng} / \mathrm{ml}$. 
Serum quantitative troponin was $0.5 \mathrm{ng} / \mathrm{ml}$ (normal lab value $0-0.6 \mathrm{ng} / \mathrm{ml}$ ). The ECG showed widespread ST-segment elevation.

The diagnosis was acute pericarditis as he fulfilled two items of ESC diagnostic criteria for pericarditis (pericardiac chest pain and new widespread ST-segment elevation) in addition to elevation in inflammatory markers. The patient received I.V fluids and Ibuprofen tabs. Due to the current COVID-19 pandemic and lymphopenia, a nasopharyngeal swab for SARS-CoV-2 was taken to be tested by reverse transcriptionpolymerase chain reaction(PCR) and the result came as positive. The patient was admitted to the isolation centre of COVID-19 and received paracetamol infusion, low molecular weight heparin (LMWH) $75 \mathrm{IU} / \mathrm{kg}$, dexamethasone $6 \mathrm{mg}$ QID, and vitamins supplement.

On the fifth day of admission, the patient developed mild shortness of breath and dry cough. On physical examination, he was dyspneic and tachypnoeic, further assessment revealed that the apex beat is difficult to locate with muffled first and second heart sound, there no added sounds or murmurs. The ECG showed low voltage in all leads. (Figure 1) The chest radiograph revealed an enlarged cardiac silhouette and with the absence of pulmonary infiltrates, and no pleural effusion was detected. (Figure 2). Subsequently, a transthoracic echocardiogram was performed which showed a moderate pericardial effusion with no regional wall motion abnormalities.(Figure 3) The LVEF was 55\%. The patient was continued on LMWH 75 IU / $\mathrm{kg}$, dexamethasone $6 \mathrm{mg}$ QID, and vitamins supplement.

\section{Discussion:}

The coronavirus infection 2019 (COVID-19), which is caused by SARS-CoV-2, causes mainly respiratory tract symptoms. While much of the focus has been on pulmonary complications, emergency clinicians need to be aware of the cardiovascular complications as well, which can be a significant contributor to the deaths associated with this disease.

Cardiovascular complications that are associated with COVID-19 include arrhythmias, acute myocardial infarction, myocarditis, heart failure and thromboembolic events.

These complications could be explained by many mechanisms such as; plaque rupture and coronary thrombosis, exaggerated immune response, direct myocardial injury, systemic inflammation, altered myocardial demand-supply ratio, electrolyte imbalances or adverse effects of therapies. ${ }^{7}$

Acute pericarditis, which is an inflammation of the sac surrounding the heart, is a rare extrapulmonary complication of COVID-19. Generally, the aetiology of pericarditis could be classified into infectious and noninfectious. ${ }^{8}$ They depend on the clinical context and epidemiological framework. In developing countries, viral infections represent the most common cause, whereas tuberculosis is the commonest cause globally.

Generally, it is postulated that viruses can cause pericardial inflammation via direct cytotoxic effects or immune-mediated mechanisms. ${ }^{9}$ COVID-19 has been stated to trigger an exaggerated systemic inflammatory response in certain patients. ${ }^{10}$ likewise in other viral infections, this inflammatory response could lead to pericarditis and the consequent pericardial effusion; however, the exact mechanism is be explained.

According to European Society of Cardiology (ESC) guidelines, the diagnosis of acute pericarditis depends on the presence of at least two out of four criteria: pericarditic chest pain, pericardial rub, new widespread ST-segment elevation or PR depression and new or worsening pericardial effusion. Elevation of inflammatory markers (white blood cell count, C-reactive protein and erythrocyte sedimentation rate), and evidence of pericardial inflammation on computed tomography (CT), cardiac magnetic resonance (CMR) can support the diagnosis of acute pericarditis.

The mainstay treatment of acute pericarditis is a high-dose aspirin and NSAIDs, other options include colchicines. A patient who has contraindications or failed to respond to first-line therapy may get benefit from corticosteroid. Using corticosteroid and NSAIDs could worsen the general condition of COVID-19 patients, which has created a management dilemma due to the concerns of the safety of using high-dose aspirin safety in these patients. ${ }^{8}$ To date, there is no convincing evidence for or against the use of high- 
dose aspirin in Covid-19 patients. ${ }^{7}$ The matters were mainly regarding the use of ibuprofen and there is no compelling evidence available connecting worsening of COVID-19 patients status with aspirin or other NSAIDs. To navigate this uncertainty, the Centre for Disease Control and Prevention (CDC) and the US Food and Drug Administration (FDA) supported the use of NSAIDs when clinically indicated. Dabbagh et al. reported the successful use of corticosteroids and colchicine combination in a patient with cardiac tamponade and COVID-19. ${ }^{3}$

Predictors of poor prognosis in acute pericarditis include the following, fever more than 38 , large pericardial effusion, subacute onset, cardiac tamponade and lack of response to NSAID or aspirin after at least one week of therapy. Therefore, the presence of fever of $38.1 \mathrm{C}$ in this patient represents one of the poor prognostic features in this attack of pericarditis.

\section{Conclusion:}

Acute pericarditis and pericardial effusion are rare extrapulmonary presentations of COVID-19, especially without concomitant pulmonary disease or myocardial injury. A high index of suspicion is necessary to assure early diagnosis and treatment. Studies are needed to determine the exact mechanism of acute pericarditis and pericardial effusion in COVID-19 patients and the optimal therapeutic strategies.

\section{Declarations:}

\section{Consent for publication:}

Written consent for publication has been obtained from the patient.

\section{Availability of data and material:}

The data used in this report is available to readers.

\section{Conflict of interests:}

The authors declare that there is no conflict of interest.

\section{Funding :}

None

\section{Acknowledgement:}

None

\section{Author Contribution :}

Waddah Osman: Diagnosed the case and participated in preparing the data.

Abdelmuniem Ahmed: Participated in preparing the data and writing the article.

Omer Elawad: Participated in preparing the data and writing the article.

Ahmed Albashir: Participated in preparing the data and writing the article.

\section{References:}

1.Fried JA, Ramasubbu K, Bhatt R, Topkara VK, Clerkin KJ, Horn E, et al. The variety of cardiovascular presentations of COVID-19. Circulation 2020 Apr 3. doi: 10.1161/CIRCULATIONAHA.120.047164 [Epub ahead of print].

2. Allam HH, Kinsara AJ, Tuaima T, Alfakih S. Pericardial Fluid in a COVID-19 Patient: Is It Exudate or Transudate?. Eur J Case Rep Intern Med . 2020;7(6):001703. Published 2020 May 12. doi:10.12890/2020_001703. 
3.Dabbagh MF, Aurora L, D'Souza P, Weinmann AJ, Bhargava P, Basir MB. Cardiac Tamponade Secondary to COVID-19 [published online ahead of print, 2020 Apr 23]. JACC Case Rep. 2020;2(9):1326-1330. doi:10.1016/j.jaccas.2020.04.009.

4.Hua A, O'Gallagher K, Sado D, Byrne J. Life-threatening cardiac tamponade complicating myo-pericarditis in COVID-19. Eur Heart J. 2020;41(22):2130. doi:10.1093/eurheartj/ehaa253.

5.Purohit R, Kanwal A, Pandit A, et al. Acute Myopericarditis with Pericardial Effusion and Cardiac Tamponade in a Patient with COVID-19. Am J Case Rep. 2020;21:e925554. Published 2020 Jul 1. doi:10.12659/AJCR.925554.

6.Asif T, Kassab K, Iskander F, Alyousef T. Acute Pericarditis and Cardiac Tamponade in a Patient with COVID-19: A Therapeutic Challenge. Eur J Case Rep Intern Med. 2020;7(6):001701. Published 2020 May 6. doi:10.12890/2020_001701.

7.Russell B, Moss C, Rigg A, Van Hemelrijck M. COVID-19 and treatment with NSAIDs and corticosteroids: should we be limiting their use in the clinical setting? Ecancermedicalscience 2020;14:1023.

8.Imazio M Spodick DH Brucato A Trinchero R Adler Y . Controversial issues in the management of pericardial diseases. Circulation2010;121:916-928.

9. Adler Y., Charron P., Imazio M. 2015 ESC Guidelines for the diagnosis and management of pericardial diseases: the Task Force for the Diagnosis and Management of Pericardial Diseases of the European Society of Cardiology (ESC) Endorsed by: The European Association for Cardio-Thoracic Surgery (EACTS) Eur Heart J. 2015;36:2921-2964.

10. Inciardi R.M., Lupi L., Zaccone G. Cardiac involvement in a patient with coronavirus disease 2019 (COVID-19) JAMA Cardiol. 2020 Mar 27.

Figure legends list:

Figure 1: The electrocardiography (ECG): it showed low voltage in all leads.Figure 2 : Chest X-ray: it showed enlargement of cardiac outline with the absence of pulmonary infiltrates.

Figure 3 : The transthoracic echocardiogram: it showed a moderate pericardial effusion with no regional wall motion abnormalities. 

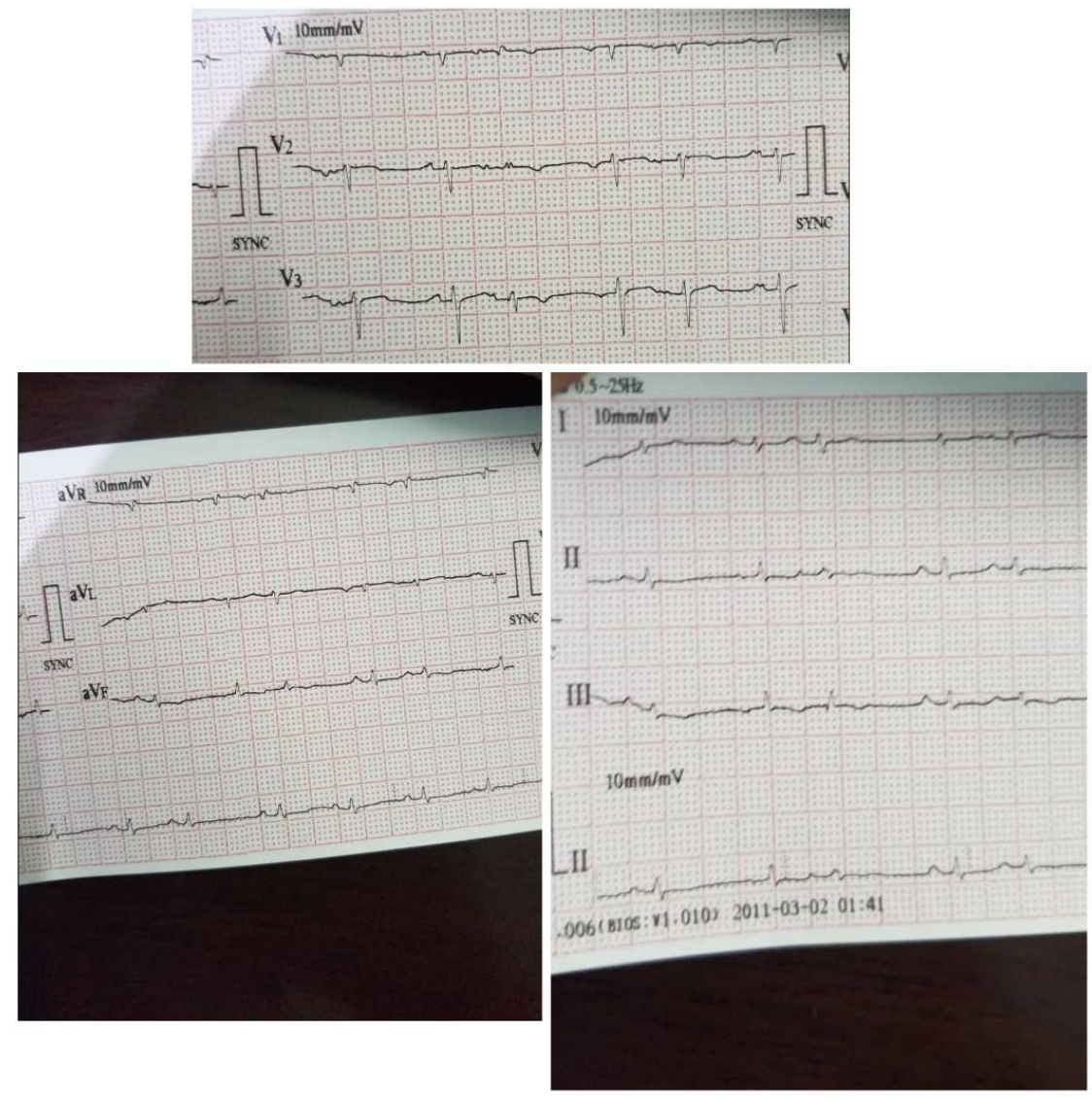

Figure 1: The electrocardiography (ECG): it showed low voltage in all leads. 


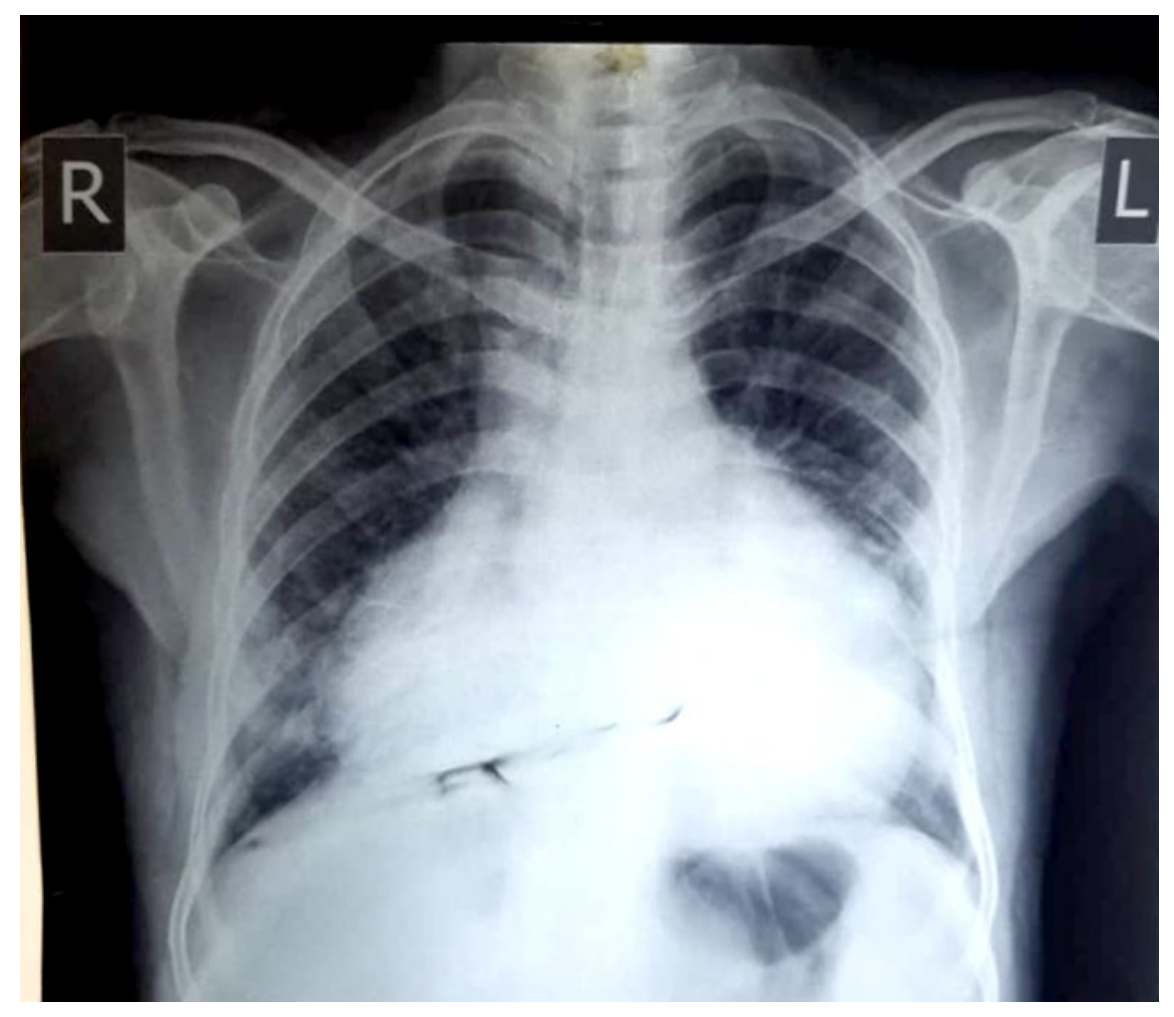

Figure 2 : Chest X-ray: it showed enlargement of cardiac outline with the absence of pulmonary infiltrates. 

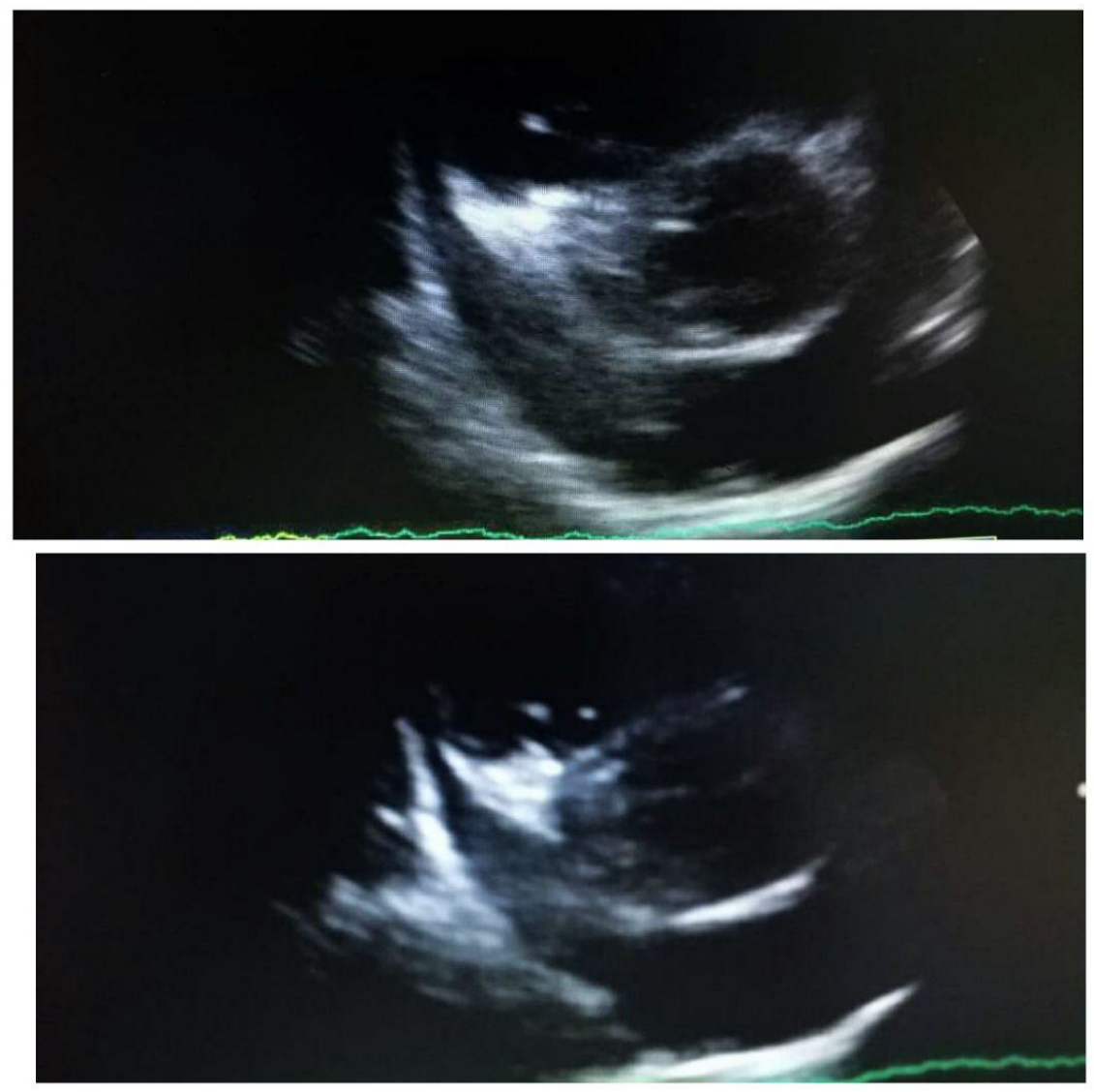

Figure 3 : The transthoracic echocardiogram: it showed a moderate pericardial effusion with no regional wall motion abnormalities 